\title{
Color Image Segmentation Based on Vectorial Multiscale Diffusion with Inter-scale Linking
}

\author{
V.B. Surya Prasath \\ Department of Mathematics, Indian Institute of Technology Madras, India \\ surya.iit@gmail.com
}

\begin{abstract}
We propose a segmentation scheme for digital color images using vectorial multiscale anisotropic diffusion. By integrating the edge information, diffusion based schemes can remove noise effectively and create fine to coarse set of images known as scale-space. Segmentation is performed by effectively tracking edges in an inter-scale manner across this scale space family of images. The regions are connected according to color coherency, and scale relation along time axis of the family is taken into account for the final segmentation result. Fast total variation diffusion and anisotropic diffusion facilitate denoising and create homogenous regions separated by strong edges. They provide a roadmap for further segmentation with persistent edges and flat regions. The scheme is general in the sense that other anisotropic diffusion schemes can be incorporated depending upon the requirement. Numerical simulations show the advantage of the proposed scheme on noisy color images.
\end{abstract}

Keywords: Image Segmentation, Multiscale diffusion, Nonlinear scale space, Color images.

\section{Introduction}

Digital image segmentation is the task of separating and identifying important objects in a scene. Segmentation techniques can be broadly classified into two categories, namely, edge based and region based approaches. Edge based segmentation methods 5,12 make use of the probable edges found as a roadmap in driving the segmenting contours. Variational minimization methods 9] and partial differential equations (PDEs) [1] based segmentation schemes are very popular in this regard. Digital images usually consists of objects of different shapes and scales. Capturing the correct local scale is an important problem in edge based image processing schemes. Thus if the objects are not differentiated with respect to their corresponding scale then the segmentation can give false results. The random noise makes it difficult to calculate the exact scale of objects, and this, in turn, gives over-segmentation or under-segmentation. In this paper we propose to tackle these problems by considering the scale space approach based on an edge preserving multiscale diffusion scheme.

Starting from the linear scale space studied in image denoising 78 nonlinear diffusion PDEs 10111 are widely used in early computer vision problems. By

S. Chaudhury et al. (Eds.): PReMI 2009, LNCS 5909, pp. 339-344, 2009.

(C) Springer-Verlag Berlin Heidelberg 2009 
using such scale space generated by them and pruning it according to inter-scale relations with region linking gives an effective segmentation of the image at the coarse scale. The edges are preserved through the scale space due to the use of multiscale diffusion and depending on the choice of scale, meaningful segmentations can be obtained. The proposed scheme is a general one with possible modifications can be made at the scale space generation step. The rest of the paper is organized as follows. Section 2 introduces the PDE based segmentation scheme. Section 3 presents numerical results which illustrate advantages of the proposed scheme on noisy images. Finally, section 4 concludes the paper.

\section{Diffusion Based Segmentation}

\subsection{Anisotropic Diffusion}

To denoise a noisy image $u_{0}$ Perona and Malik proposed the following anisotropic diffusion scheme (ADS) [10]

$$
\frac{\partial u(x, t)}{\partial t}=\operatorname{div}(g(|\nabla u(x, t)|) \nabla u(x, t)) \quad \text { with } \quad u(x, 0)=u_{0}
$$

where $g$ is a decreasing function, for example $g(s)=\exp -\left(s^{2} / K\right), K>0$ is contrast parameter. The main idea here is to control the diffusion near edges and smooth the flat regions, thereby integrating edge preserving nature into the diffusion process. The ADS (11) creates the scale space of images $\{u(x, t)\}_{t=0}^{T}$ with $T$ as the final scale of choice, see Figure 1 Total variation (TV) function $g(s)=s^{-1}$ based diffusion PDE is proven to be more effective [2] than the original formulation (11) and it preserves edges across multiple scales better. TV based PDE is given by

$$
\frac{\partial u(x, t)}{\partial t}=\operatorname{div}\left(\frac{\nabla u(x, t)}{|\nabla u(x, t)|}\right) \quad \text { with } u(x, 0)=u_{0}
$$

As a first step in our segmentation algorithm we run TV scheme (2) to get rid of of the noise present and create a family of images based on the scale parameter $t$, see Figure 1(a).

\subsection{Segmentation Scheme}

Motivated by studies on scale space [10778 and region extraction methods we prune the scale space created from the nonlinear diffusion (11) using color distances and coherent linking of objects along the scale axis. Since CIE-La*b* color space offers more perceptually uniform color distances with $L^{2}$ distance metric, $\|u-v\|^{2}:=\left(u_{L}-v_{L}\right)^{2}+\left(u_{a *}-v_{a *}\right)^{2}+\left(u_{b *}-v_{b *}\right)^{2}$, we make use of it in our algorithm. Our segmentation scheme is based on a key observation: as one traverse across the compact scale space $\{u(x, t)\}_{t=0}^{T}$, the local and coarse scale information is connected to a fine scale information progressively. 


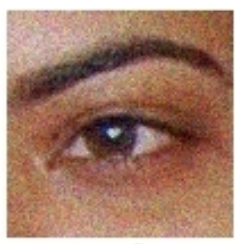

$t=0$

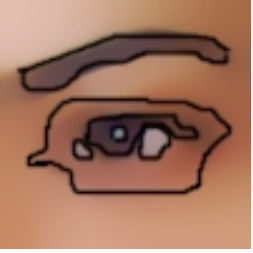

(b) $\left\{\mathcal{C}_{300}^{r}\right\}_{r=0}^{6}$

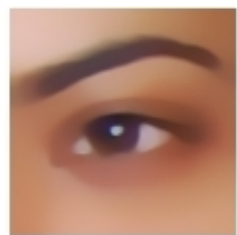

$t=100$

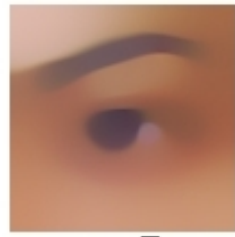

$\mathrm{t}=\mathrm{T}$

(a) $\{u(x, t)\}_{t=0}^{T}$

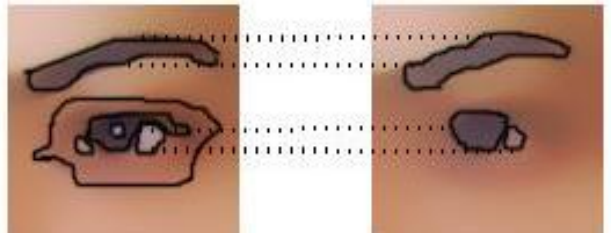

(c)

Fig. 1. (a) Scale space, $T=500$ (b) Intra-scale splitting, shown at $t=300$ (c) Interscale linking, shown for $\mathcal{C}_{300}^{r} \rightarrow \mathcal{C}_{400}^{k}$

(1) Intra-scale splitting: We partition the image domain $\mathcal{D}_{t}$ of $u(x, t)$ by connected components $\left\{\mathcal{C}_{t}^{r}\right\}_{r=0}^{P_{t}}$ where $P_{t}$ the total number of distinct regions at scale $t$, in the following way:

$$
\mathcal{C}_{t}^{r}:=\left\{x \in D_{t}: u(x, t)=\text { const }\right\} \quad \& \quad \mathcal{C}_{t}^{r} \bigcap \mathcal{C}_{t}^{l}=\emptyset, \quad \mathcal{D}_{t}=\bigcup_{r=0}^{P_{t}} \mathcal{C}_{t}^{r}
$$

Note that $\left\{\mathcal{C}_{t}^{r}\right\}$ are the level lines of the image $u(x, t)$ (see Figure 2(a) and (b)) and by [4] we know that the geometry of a color image is entirely described by them. Hence this splitting respects image boundaries through diffused images.

(2) Inter-scale linking step: Next we link two subsequent scale regions (say $t$ and $t+1$ ) using the minimum color distance and overlapping as follows:

$$
\mathcal{L}\left(\mathcal{C}_{t}^{r}\right)=\left\{\min _{\mathcal{C}_{t+1}^{r}}\left\|u\left(\mathcal{C}_{t+1}^{k}, t+1\right)-u\left(\mathcal{C}_{t}^{r}, t\right)\right\|: \mathcal{C}_{t+1}^{k} \bigcap \mathcal{C}_{t}^{r} \neq \emptyset\right\}
$$

and we denote it by $\mathcal{C}_{t}^{r} \rightarrow \mathcal{C}_{t+1}^{k}$. This can be iterated among scales and in general one can obtain $\mathcal{C}_{t_{1}}^{r} \rightarrow \mathcal{C}_{t_{2}}^{k}$ for $t_{1}<t_{2}$ (see Figure 1(c)).

(3) Final merging step: All connected regions $\left\{\mathcal{C}_{0}^{r}\right\}_{t=0}^{P_{0}}$ of the initial image $u(x, 0)$ that are linked to the same region at the end scale $T$ are coming from one object. Thus the final segmentation can be obtained at scale level $T$ as

$$
\mathcal{S}_{T}^{k}=\bigcup_{r=0}^{P_{0}} \mathcal{C}_{0}^{r} \quad \& \quad \mathcal{C}_{0}^{r} \rightarrow \mathcal{C}_{T}^{k}
$$


where $\left\{\mathcal{S}_{T}^{k}\right\}_{r=0}^{P_{T}}$ are the segmented objects and $P_{T}$ is the number of segmented objects.

The above methodology effectively combines the edge preserving property of nonlinear scale space based on multiscale anisotropic diffusion (1) with hierarchical segmentation and scale tracking.

\section{$3 \quad$ Numerical Results}

All the images in the computations are normalized to $[0,1]$ and MATLAB ${ }^{\circledR} 7.4$ is used for visualization purposes. To compute color distances in the inter-scale linking step we convert the image from RGB to CIE-La*b* using the D65 white point reference. Recently, Bresson and Chan [2] formulated a fast algorithm for vectorial version of TV (2) using duality arguments. In our experiments we make use of it for its computational efficiency and the edge preserving property of TV diffusion. The scheme takes about 80 secs for an image of size $256 \times 256$ to run on a Pentium IV $2.40 \mathrm{GHz}$ PC with 20 secs for diffusion (100 iterations) and the remaining time for the segmentation scheme. An additive Gaussian noise of $\sigma=20$ is added to the original test images in all the experiments. Figure 2 compares the TV based (2) and ADS scheme (1) based segmentation results. As can be seen by comparing the corresponding contour maps

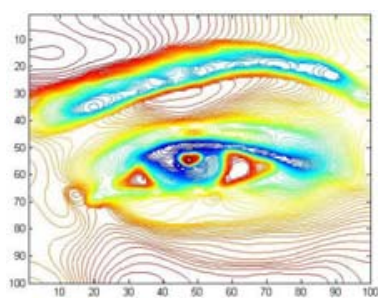

(a)

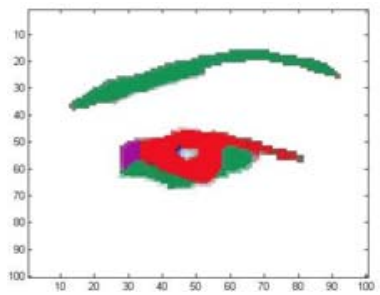

(c)

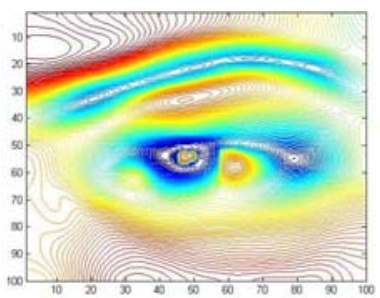

(b)

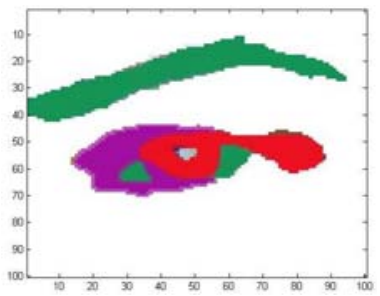

(d)

Fig. 2. (a) Level lines of the L channel for $u^{100}$ using the TV flow (2) (b) Level lines of the L channel for $u^{100}$ using the ADS with $\left(g(s)=\exp -\left(s^{2} / 20\right)\right)$ in (1) (c) Segmented image based on TV flow and (d) Segmented image based on ADS 
(Figure2(a) and (b)), TV scheme segmentation (Figure 2(c)) yields a better segmentation in terms of the pupil and the eyebrow than ADS scheme segmentation (Figure 2(d)).

\subsection{Comparison Results}

We compare our scale space based segmentation scheme with the following schemes: linear scale space based scheme [7] (setting $g \equiv 1$ in (11)), geodesic active contours 5], Level set based scheme [12] (based on Mumford-Shah functional), Graph based scheme [6] (bottom-up clustering). Figure 3] shows the visual comparison results on a noisy Peppers color image. We use the same colors for segments in all the images to show the effect. As can be seen our scheme using TV scheme (2) (Figure 3(f)) results in a better segmentation of the middle pepper without any artifacts.

We used the CIE-La*b* space and $L^{2}$ metric for inter-scale linking step, other choices are also possible, for example $H S V$ and chrominance-brightness space. Also adaptive selection of stopping time $T$ [11] (also known as scale selection [38] in scale space literature) for the diffusion scheme (11) involved, determines the level of segmentation, and it remains to be explored. Comparing with other region based segmentation approaches quantitatively and the use of other anisotropic diffusion PDE based schemes [1] (for example, chromatic diffusion) defines our future work in this direction.

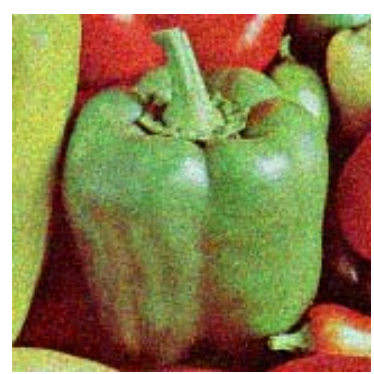

(a) Input

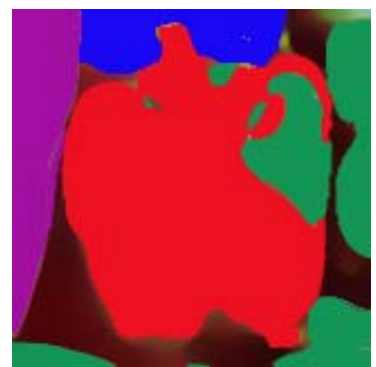

(d) Level set 12

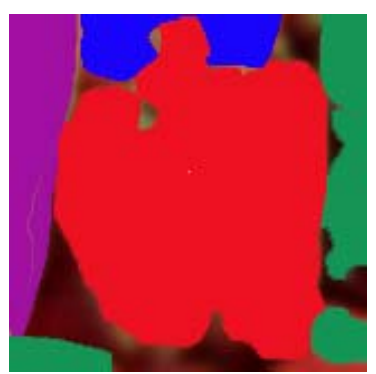

(b) Linear [7]

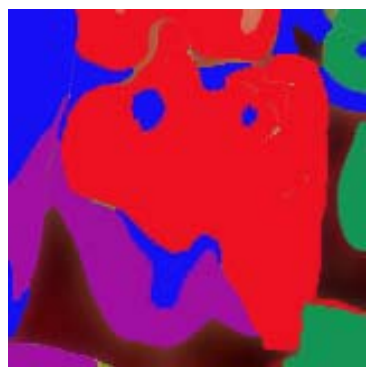

(e) Graph 6]

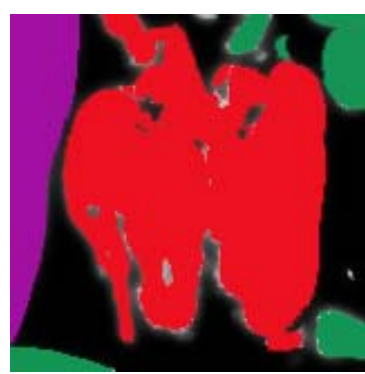

(c) Active contours [5]

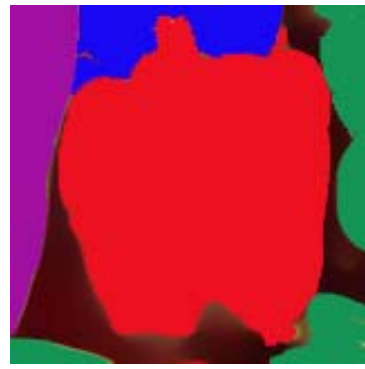

(f) TV-Pruning

Fig. 3. Comparison of segmentation results on Peppers image 


\section{Conclusions}

We used the nonlinear scale space concept to segment color images. The continuous family of images generated from nonlinear diffusion PDE are arranged according to their increasing scale and pruned using a track and merge scheme. Multiscale diffusion captures the edges without spurious oscillations and aides in scale capturing at the final scale of choice. By utilizing the fine to coarse set of images the segmentation is done iteratively. Preliminary numerical examples on images and comparison with related schemes show that the proposed approach gives better segmentation result on noisy and real images.

\section{References}

1. Aubert, G., Kornprobst, P.: Mathematical problems in image processing: Partial differential equations and the calculus of variations. Applied Mathematical Sciences, vol. 147. Springer, New York (2006)

2. Bresson, X., Chan, T.F.: Fast dual minimization of the vectorial total variation norm and applications to color image processing. Inverse Problems and Imaging 2(4), 455-484 (2008)

3. Brox, T., Weickert, J.: A TV flow based local scale measure for texture discrimination. In: Pajdla, T., Matas, J.G. (eds.) ECCV 2004. LNCS, vol. 3022, pp. 578-590. Springer, Heidelberg (2004)

4. Caselles, V., Coll, B., Morel, J.-M.: Geometry and Color in Natural Images. J. Math. Imag. Vis. 16, 89-105 (2002)

5. Caselles, V., Kimmel, R., Sapiro, G.: Geodesic active contours. Int. J. Comput. Vis. 22(1), 61-79 (1997)

6. Felzenszwalb, P.F., Huttenlocher, D.P.: Efficient Graph-Based Image Segmentation. Int. J. Comput. Vis. 59(2), 167-181 (2004)

7. Florack, L.M., ter Haar Romeny, B.M., Koenderink, J.J., Viergever, A.M.: Linear scale-space. J. Math. Imag. Vis. 4(4), 325-351 (1994)

8. Lindeberg, T.: Edge detection and ridge detection with automatic scale selection. Int. J. Comput. Vis. 30(2), 117-156 (1998)

9. Morel, J.-M., Solimini, S.: Variational Methods in Image Processing. Birkhauser, Boston (1994)

10. Perona, P., Malik, J.: Scale-space and edge detection using anisotropic diffusion. IEEE Trans. Pattern Anal. Machine Intell. 12(7), 629-639 (1990)

11. Vanhamel, I., Mihai, C., Sahli, H., Katartzis, A., Pratikakis, I.: Scale selection for compact scale-space representation of vector-valued images. Int. J. Comput. Vis. 84(2), 194-204 (2009)

12. Vese, L.A., Chan, T.F.: A multiphase level set framework for image segmentation using the Mumford Shah model. Int. J. Computer Vis. 50(3), 271-293 (2002) 